\title{
Endoscopic Ultrasound-Guided Drainage of Peripancreatic Fluid Collections
}

\author{
Eun Young Kim ${ }^{1}$ and Robert H. Hawes ${ }^{2}$ \\ ${ }^{1}$ Department of Internal Medicine, Catholic University of Daegu School of Medicine, Daegu, Korea, ${ }^{2}$ Center for Interventional Endoscopy, \\ AdventHealth Orlando, Orlando, FL, USA
}

See "Comparison of Clinical Outcomes between Plastic Stent and Novel Lumen-apposing Metal Stent for Endoscopic UltrasoundGuided Drainage of Peripancreatic Fluid Collections” by Ho Cheol Shin, Chang Min Cho, Min Kyu Jung, et al., on page 353-359.

The 2012 revised Atlanta classification defines pancreatic fluid collections (PFCs) as acute peripancreatic fluid collections, acute necrotic collections, pseudocysts, or walled-off necroses (WONs). ${ }^{1}$ Pseudocysts and WONs have an organized wall around the collection, which usually develops at least 4 weeks after the onset of acute pancreatitis. Pancreatic pseudocysts and WONs can also occur in patients with chronic pancreatitis by an acute exacerbation of pancreatitis or by progressive ductal obstruction. Drainage of pancreatic pseudocysts or WONs is necessary in patients who are symptomatic or have biliary or intestinal obstruction or if the size of the collection increases rapidly.

Endoscopic drainage is an accepted alternative to surgical or radiology-guided percutaneous drainage when intervention is indicated. One recent single-center randomized trial showed that endoscopic drainage for infected necrotizing pancreatitis significantly reduces major complications, lowers costs, and increases quality of life compared with minimally invasive surgery. ${ }^{2}$ Endoscopic transmural drainage can be performed by conventional endoscopy or endoscopic ultrasound (EUS).

Received: July 8, 2019 Accepted: July 10, 2019

Correspondence: Robert H. Hawes

Center for Interventional Endoscopy, AdventHealth Orlando, 601 E Rollins St, Orlando, FL 32803, USA

Tel: +1-407-609-3355, Fax: +1-407-303-0795, E-mail: Robert.Hawes.MD@AdventHealth.com

ORCID: https://orcid.org/0000-0003-2709-8530

(c) This is an Open Access article distributed under the terms of the Creative Commons Attribution Non-Commercial License (http://creativecommons.org/ licenses/by-nc/3.0) which permits unrestricted non-commercial use, distribution, and reproduction in any medium, provided the original work is properly cited.
EUS-guided drainage of PFCs is now the standard of care because it enables identification of intervening vasculature, assessment of the distance between the lumen and PFC, and makes the procedure possible when definitive luminal compression is not endoscopically visible.

In this issue of Clinical Endoscopy, Shin et al. ${ }^{3}$ present a retrospective study comparing the clinical outcome of EUS-guided drainage of PFCs between using a plastic stent (PS) and lumen-apposing metal stent (LAMS). The technical success rate was similar in both groups $(94.1 \%$ vs. $100 \%, p=1.0)$, but the procedure time was significantly shorter in the LAMS group (10.6 $\pm 2.5 \mathrm{~min}$ vs. $21.4 \pm 9.5 \mathrm{~min}, p=0.002$ ). Clinical success was achieved in all patients with technical success, but recurrence of PFCs after stent removal occurred in $41.7 \%$ and $40.0 \%$ $(p=1.0)$ in the PS and LAMS groups, respectively.

One should be cautious while interpreting the results from this study because it was retrospective in design, involved a relatively small number of patients, and was conducted over a 7-year period (from January 2011 to December 2017). EUS-guided transmural drainage of PFCs with PSs was performed in the initial period of procedure development (17 patients between January 2011 and October 2016), whereas the 10 LAMS procedures (SPAXUS ${ }^{\circledR}$; Taewoong Medical, Seoul, Korea) were performed between October 2016 to December 2017. This also highlights the difference in workload; the 17 PS procedures were completed over a 6-year period (3 patients per year), whereas the 10 LAMS procedures were all done in 1 year. The reported recurrence rate of PFCs after successful drainage by EUS-guided transmural drainage varies in the 
literature, but averages $10 \%-15 \%$, which is significantly lower than the $40 \%$ rate noted in this study. This is likely related to the incidence of disconnected pancreatic duct syndrome (DPDS), which may have been higher in this study. Finally, all 17 PFCs in the PS group were pseudocysts, whereas in the LAMS group, 8 were pseudocysts and 2 were WONs. Treatment success is more likely in patients with pseudocysts than in those with WONs. ${ }^{4}$

One systematic review conducted in 2014 involving 17 studies, which compared the efficacy of endoscopic transmural drainage for PFCs, found no differences in the treatment success rate (81\% [95\% confidence interval (CI), 77\%-84\%] vs. $82 \%$ [95\% CI, 74\%-88\%]), adverse events rate (16\% [95\% CI, $14 \%-39 \%$ ] vs. $23 \%$ [95\% CI, $16 \%-33 \%]$ ), and recurrence rate (10\% [95\% CI, 8\%-13\%] vs. 9\% [95\% CI, 4\%-19\%]) between the plastic and metal stents. The treatment success rates were $85 \%$ (95\% CI, $81 \%-89 \%$ ) vs. $83 \%$ (95\% CI, $74 \%-89 \%$ ) for pseudocysts and 70\% (95\% CI, 62\%-76\%) vs. 78\% (95\% CI, 50\%-93\%) for WONs with the plastic and metal stents, respectively. ${ }^{5}$

Recent data suggest that the bleeding risk with LAMSs may be higher when compared with double-pigtail PSs. A report from a tertiary care center showed a bleeding risk of $4 \%$ in the LAMS group (10 pseudocysts and $9 \mathrm{WONs}$ ) vs. $1 \%$ in the double-pigtail PS group (70 pseudocysts and $14 \mathrm{WONs}){ }^{6}$ In one study, the rates of bleeding were $7 \%$ vs. $2 \%$ in the LAMS and PS groups, respectively, when 313 WON patients were treated with EUS-guided stent placement and debridement (86 patients with LAMSs, 106 patients with double-pigtail PSs, and 121 patients with fully-covered self-expandable metal stents [FCSEMSs]). ${ }^{7}$ No bleeding case was noted in the FCSEMS group in that report. However, biliary FCSEMSs are no longer used because of the high stent migration rate. LAMSs, such as the $\operatorname{AXIOS}^{\circledR}$ (Boston Scientific, Marlborough, MA, USA), Nagi $^{\circledR}$ (Taewoong Medical), or Spaxus ${ }^{\circledR}$ (Taewoong Medical) stents, which are designed with wide flanges on both ends to prevent migration, have a relatively wide diameter and short length. Following the placement of LAMSs with large luminal diameter, endoscopic necrosectomy can be performed after exchanging the EUS scope for a gastroscope. ${ }^{8}$ One potential advantage of LAMSs is that fewer procedures are required to achieve resolution of WONs. When WONs contain debris, additional placement of a nasocystic tube in the LAMSs may improve the clinical success. In a retrospective study of 87 patients with WONs that contained solid debris, patients with both transmural stents and a nasocystic tube were more likely to have successful drainage than those with only transmural stents at the 1-year follow-up $(p=0.059){ }^{9}$ However, whether a combination of endoscopic necrosectomy was performed or not was undescribed in that report.
The decision of whether to use LAMSs or PSs to drain PFCs must be individualized. In part, it depends on the nature of the PFC. In the case of pseudocysts, the overall success rates are the same and are greater than $90 \%$ with both techniques. PSs are less expensive, but LAMS procedures are easier to perform and are less time-consuming. The case of WONs is more complex and beyond the scope of this editorial. The one issue that should be emphasized is that in the case of DPDSs, long-term treatment to avoid recurrence must involve the use of PSs. ${ }^{10}$ EUS-guided drainage of PFCs has become popular nowadays. Further adoption is expected with the development of better accessories and stents resulting in ease of implementation, speedy use, and lower complication rates.

\section{Conflicts of Interest}

Robert H. Hawes receives consulting fees from Boston Scientific Corporation, but not in the area of EUS-guided drainage of PFCs. Other author has no financial conflicts of interest.

\section{REFERENCES}

1. Banks PA, Bollen TL, Dervenis C, et al. Classification of acute pancreatitis--2012: revision of the Atlanta classification and definitions by international consensus. Gut 2013;62:102-111.

2. Bang JY, Arnoletti JP, Holt BA, et al. An endoscopic transluminal approach, compared with minimally invasive surgery, reduces complications and costs for patients with necrotizing pancreatitis. Gastroenterology 2019;156:1027-1040.e3.

3. Shin HC, Cho CM, Jung MK, Yeo SJ. Comparison of clinical outcomes between plastic stent and novel lumen-apposing metal stent for endoscopic ultrasound-guided drainage of peripancreatic fluid collections. Clin Endosc 2019;52:353-359.

4. Varadarajulu S, Bang JY, Phadnis MA, Christein JD, Wilcox CM. Endoscopic transmural drainage of peripancreatic fluid collections: outcomes and predictors of treatment success in 211 consecutive patients. J Gastrointest Surg 2011;15:2080-2088.

5. Bang JY, Hawes R, Bartolucci A, Varadarajulu S. Efficacy of metal and plastic stents for transmural drainage of pancreatic fluid collections: a systematic review. Dig Endosc 2015;27:486-498.

6. Lang GD, Fritz C, Bhat T, et al. EUS-guided drainage of peripancreatic fluid collections with lumen-apposing metal stents and plastic double-pigtail stents: comparison of efficacy and adverse event rates. Gastrointest Endosc 2018;87:150-157.

7. Siddiqui AA, Kowalski TE, Loren DE, et al. Fully covered self-expanding metal stents versus lumen-apposing fully covered self-expanding metal stent versus plastic stents for endoscopic drainage of pancreatic walled-off necrosis: clinical outcomes and success. Gastrointest Endosc 2017;85:758-765.

8. Alali A, Mosko J, May G, Teshima C. Endoscopic ultrasound-guided management of pancreatic fluid collections: update and review of the literature. Clin Endosc 2017;50:117-125.

9. Siddiqui AA, Dewitt JM, Strongin A, et al. Outcomes of EUS-guided drainage of debris-containing pancreatic pseudocysts by using combined endoprosthesis and a nasocystic drain. Gastrointest Endosc 2013;78:589-595.

10. Park SW. Is endoscopic ultrasound-guided drainage alone sufficient for the treatment of peripancreatic fluid collection? Clin Endosc 2017;50:316-317. 